\title{
Phase transitions and dynamical properties of quasi-one-dimensional structures with hydrogen bonds
}

\author{
R.Ya. Stetsiv \\ Institute for Condensed Matter Physics of the National Academy of Sciences of Ukraine, \\ 1 Svientsitskii Str., 79011 Lviv, Ukraine
}

Received November 7, 2011, in final form January 26, 2012

\begin{abstract}
The frequency dependence of dynamical conductivity of the quasi-one-dimensional structures with hydrogen bonds is studied on the basis of pseudospin-electron model. It takes into account the proton-electron interaction, external longitudinal field $h$, the tunneling hopping of protons, electron transfer and direct interaction between protons. The dependences of the electron concentration and mean number of protons at the site on temperature and external field are obtained. The phase transition lines from uniform phase into charge ordered phase are determined. The dependence of dynamical conductivity on temperature and field $h$ and its changes at the phase transitions are obtained.
\end{abstract}

Key words: pseudospin-electron model, proton-electron interaction, hydrogen bonds, conductivity

PACS: $72.60 .+g, 36.40 . c$

\section{Introduction}

The properties of molecular and crystalline structures with hydrogen bonds are mainly determined by the character of proton redistribution on the bonds. We investigate the microscopic mechanisms of charge transfer in such systems on the basis of the proposed pseudospin-electron model [1, 2] that takes into account the correlation between the proton displacement and reconstruction of electron states as well as the change of their occupancy. This interaction manifests itself as a cooperative proton-electron transfer (PET) in a number of experimental works [3-10] and it follows also from the results of quantumchemical calculations [1, 11-13]. Quantum chemical methods allow us to examine these charge redistributions more in detail. The structural and optical studies of the proton transfer in $N$-salicylideneaniline [8, 10] show that photochromism and thermochromism in these object arise from a proton transfer that is accompanied by a configurational change of electron structure. It was shown that the behaviour of proton dynamics is quite consistent with the temperature dependence of visible absorption spectra of this crystal. If we could construct a molecular conductor based on this type of molecules, the charge transport might strongly be modulated by the proton motion. Photoinduced proton-coupled electron transfer (PCET) is investigated in a number of works [14-17] as one of the mechanisms of energy transformation in biological and chemical systems. The effect of a such proton-electron coupling plays an important role in passing a proton through the biological membrane in photosynthesis. The design of an electron-proton hybrid system using the elements of one-dimensional metal chains, acceptor (or donor) molecules, and interchain H-bonds are proposed [4]. A new molecular function is expected to be produced in this system, if the motion of proton is closely correlated with the dynamics of the 1D electronic states. A similar effect is observed in the halogen (X)-bridge mixed-valence transition-metal (M) complexes (M-X-complexes) [3]. The $\mathrm{M}$-X-complexes $\left[\mathrm{MA}_{2} \mathrm{X}\right] \mathrm{Y}_{2}(\mathrm{M}=\mathrm{Pt}$, $\mathrm{Pd}$ or $\mathrm{Ni})$ have a one-dimensional (1D) chain structure and adjacent chains are connected by hydrogen bonds. Here $\mathrm{X}$ stands for a bridging halogen ion $(\mathrm{X}=\mathrm{Cl}, \mathrm{Br}$ or J), A for a ligand molecule (e.g. ethylenediamine, cyclohexenediamine), and $\mathrm{Y}$ for a counter anion (e.g. 
$\mathrm{Y}=\mathrm{Br}^{-}, \mathrm{ClO}_{4}^{-}$). The location of the protons on $\mathrm{N}-\mathrm{H}-\mathrm{Y}$ induce additional electron charges on the ions $\mathrm{M}$ and at some conditions they form a charge-ordered state (CDW) [3]. It is pointed out that the electron-proton coupling is capable of controlling the CDW state [18].

Pseudospin-electron model was originally proposed to describe the correlated proton-electron charge transfer in a single complex with hydrogen bond [1]. This model was later extended to the description of the charge transfer in the above mentioned quasi-one-dimensional structures with hydrogen bonds. We examined uniform phases [2]. In this work we investigate the phase transition from uniform phase into charge-ordered phase in such systems. We study thermodynamic properties and the frequency dependence of dynamical conductivity and its changes at the phase transitions.

\section{Hamiltonian}

The Hamiltonian of quasi-one-dimensional structures which contains chains with hydrogen bonds are written down in the form [2]:

$$
\begin{aligned}
H_{\mathrm{eff}}= & \sum_{l} \sum_{i, \sigma}\left\{(\varepsilon-\mu) n_{i \sigma}(l)+g\left[n_{i \sigma}(l)-n_{i+1, \sigma}(l)\right] S_{i}^{z}(l)\right\} \\
& +\sum_{l, l^{\prime}} \sum_{i, j, \sigma} t_{i(l), j\left(l^{\prime}\right)}\left[a_{i \sigma}^{+}(l) a_{j, \sigma}\left(l^{\prime}\right)+a_{j, \sigma}^{+}\left(l^{\prime}\right) a_{i \sigma}(l)\right] \\
& +\sum_{l} \sum_{i} \Omega S_{i}^{x}(l) \\
& -\sum_{l} \sum_{i} h S_{i}^{z}(l) \\
& -\frac{1}{2} \sum_{l, l^{\prime}} \sum_{i, j} J_{i(l), j\left(l^{\prime}\right)} S_{i}^{z}(l) S_{j}^{z}\left(l^{\prime}\right) .
\end{aligned}
$$

Here, the summation along the chains (indices $i, j$ ) and the summation over the chains (indices $l, l^{\prime}$ ) is performed. Pseudospin operator $\hat{S}_{i}$ describes the proton position in double potential well on the hydrogen bond. We suppose that the transfer along hydrogen bond is dominant: $t=t_{i(l), i+1(l)} ; n_{i \sigma}$ is operator of electron concentration at $i$ lattice site, $\sigma$ is electron spin, $\mu$ is chemical potential of electrons.

The Hamiltonian includes proton-electron interaction (parameter $g$ ), electron transfer (parameter $t$ ), energy of proton tunneling (parameter $\Omega$ ), asymmetry of the local anharmonic potential (parameter $h$ ). The last term describes proton-proton interaction.

Pseudospin-electron interaction leads to the effective interaction between pseudospins (between protons in our case) and as it is shown in [19-21] it can cause the appearance of a modulated phase with doubling of the initial lattice period and can lead to the corresponding charge modulation. The study of this phenomenon is the aim of this paper. In a case of double modulation of the lattice period, the crystal is divided into two sublattices. We introduce the following notations: $\eta_{\alpha}=\left\langle S_{i, \alpha}^{z}\right\rangle, n_{\alpha}=\left\langle\sum_{\sigma} n_{i, \alpha, \sigma}\right\rangle,(\alpha=1,2$ is the sublattice index). In the mean field approximation (MF) and after passing to $k$-representation the Hamiltonian (1) has a form:

$$
\begin{gathered}
H_{\mathrm{MF}}=H_{\mathrm{el}}+H_{\mathrm{sp}}+U, \\
H_{\mathrm{el}}=\sum_{k, \alpha, \sigma}\left[\varepsilon-\mu+g\left(\eta_{\alpha}-\eta_{\beta}\right)\right] n_{k, \alpha, \sigma}+\sum_{k, \alpha, \sigma} t_{k, \alpha, \sigma} a_{k, \alpha, \sigma}^{+} a_{k, \beta, \sigma}, \quad \alpha \neq \beta, \\
t_{k}^{11}=t_{k}^{22}=0, \quad t_{k} \equiv t_{k}^{12}=t_{k}^{21}=\sum_{i(l)-j\left(l^{\prime}\right)} t_{i(l), j\left(l^{\prime}\right)}^{12} \exp \left\{\mathrm{i} \vec{k}\left[\vec{R}_{i(l), 1}-\vec{R}_{j\left(l^{\prime}\right), 2}\right]\right\}, \\
H_{\mathrm{sp}}=\sum_{l} \sum_{i, \alpha}\left\{\Omega S_{i, \alpha}^{x}(l)-\left[h+j \eta_{\beta}-g\left(n_{\alpha}-n_{\beta}\right)\right] S_{i, \alpha}^{z}(l)\right\}, \\
U=\frac{1}{2} N j \eta_{1} \eta_{2}-\frac{N}{2} g\left(n_{1}-n_{2}\right)\left(\eta_{1}-\eta_{2}\right), \quad J=\sum_{l^{\prime}} \sum_{j} J_{i(l), j\left(l^{\prime}\right) .}
\end{gathered}
$$


The electronic part of Hamiltonian (2) is diagonalized by unitary transformation

$$
\begin{gathered}
a_{k, 1, \sigma}=\tilde{a}_{k, 1, \sigma} \cos \varphi+\tilde{a}_{k, 2, \sigma} \sin \varphi, \\
a_{k, 2, \sigma}=-\tilde{a}_{k, 1, \sigma} \sin \varphi+\tilde{a}_{k, 2, \sigma} \cos \varphi \\
\cos 2 \varphi=\frac{-g\left(\eta_{1}-\eta_{2}\right)}{\sqrt{g^{2}\left(\eta_{1}-\eta_{2}\right)^{2}+t_{k}^{2}}}, \quad \sin 2 \varphi=\frac{t_{k}}{\sqrt{g^{2}\left(\eta_{1}-\eta_{2}\right)^{2}+t_{k}^{2}}} .
\end{gathered}
$$

Thus, we obtain:

$$
\begin{gathered}
H_{\mathrm{el}}=\sum_{k, \alpha, \sigma}\left(E_{k, \alpha}-\mu\right) \tilde{n}_{k, \alpha, \sigma}, \\
E_{k, \alpha}=\varepsilon+(-1)^{\alpha} \sqrt{g^{2}\left(\eta_{1}-\eta_{2}\right)^{2}+t_{k}^{2}} .
\end{gathered}
$$

The spin part of Hamiltonian is diagonalized by unitary transformation:

$$
\begin{aligned}
S_{i}^{x}(l) & =\tilde{S}_{i}^{x}(l) \cos \psi+\tilde{S}_{i}^{z}(l) \sin \psi, \\
S_{i}^{z}(l) & =-\tilde{S}_{i}^{x}(l) \sin \psi+\tilde{S}_{i}^{z}(l) \cos \psi, \\
\cos \psi_{\alpha} & =\left[h+J \eta_{\beta}-g\left(n_{\alpha}-n_{\beta}\right)\right] / \lambda_{\alpha}, \sin \psi_{\alpha}=\Omega / \lambda_{\alpha} .
\end{aligned}
$$

In this case:

$$
\begin{gathered}
H_{\mathrm{sp}}=-\sum_{l} \sum_{i, \alpha} \lambda_{\alpha} \tilde{S}_{i, \alpha}^{z}(l), \\
\lambda_{\alpha}=\sqrt{\left[h+J \eta_{\beta}-g\left(n_{\alpha}-n_{\beta}\right)\right]^{2}+\Omega^{2}} .
\end{gathered}
$$

\section{Thermodynamic properties}

Using formulae (2)-(5), we can write the equations for electron concentration $n_{\alpha}$ and the average mean of pseudospins $\eta_{\alpha}$ in sublattices:

$$
\begin{aligned}
n_{\alpha}= & \frac{1}{\frac{N}{2}} \sum_{k, \alpha, \sigma}\left(\frac{1+\cos 2 \varphi}{2}\left\{1+\exp \left[\beta\left(E_{k, \alpha}-\mu\right)\right]\right\}^{-1}\right. \\
& \left.+\frac{1-\cos 2 \varphi}{2}\left\{1+\exp \left[\beta\left(E_{k, \beta}-\mu\right)\right]\right\}^{-1}\right) \\
\eta_{\alpha}= & \frac{h+J \eta_{\beta}-g\left(n_{\alpha}-n_{\beta}\right)}{2 \lambda_{\alpha}} \tanh \left(\frac{\lambda_{\alpha}}{2 k T}\right)
\end{aligned}
$$

From all the possible solutions of equations (6)-7) we choose the ones that correspond to the minimum of grand canonical potential $\Phi$ in regime of $\mu=$ const or minimum of free energy $F=\Phi+\mu N$ in regime $n=$ const. In the MF-approximation:

$$
\begin{aligned}
\Phi= & -2 k T \sum_{k} \ln \left(\left\{1+\exp \left[-\beta\left(E_{k, 1}-\mu\right)\right]\right\}\left\{1+\exp \left[-\beta\left(E_{k, 2}-\mu\right)\right]\right\}\right) \\
& -\frac{1}{2} k T N \ln \left[4 \cosh \left(\frac{\lambda_{1}}{2 k T}\right) \cosh \left(\frac{\lambda_{2}}{2 k T}\right)\right] \\
& +\frac{1}{2} N J \eta_{1} \eta_{2}-\frac{1}{2} N g\left(n_{1}-n_{2}\right)\left(\eta_{1}-\eta_{2}\right) .
\end{aligned}
$$

From the relations (6)-(7) we obtain the equations for $\delta n=n_{1}-n_{2}$ and $\delta \eta=\eta_{1}-\eta_{2}$, which can play a role of the order parameter for a modulated phase. Using these equations we obtain the condition of the 
appearance of nonzero solutions for $\delta n$ and $\delta \eta$, and the equation for temperature of the second order phase transition to the modulated phase.

$$
\begin{aligned}
\frac{2}{\lambda^{2}}[ & \left.\frac{\Omega^{2}}{\lambda}\left\langle\sigma^{z}\right\rangle+\frac{1}{k T}(h+J \eta)^{2}\left(\frac{1}{4}-\left\langle\sigma^{z}\right\rangle^{2}\right)\right] \\
& \times\left[\frac{1}{2} J-\frac{4}{N} \sum_{k} \frac{g^{2}}{t_{k}} \delta \eta\left(\left\{1+\exp \left[\beta\left(\varepsilon-\left|t_{k}\right|-\mu\right)\right]\right\}^{-1}-\left\{1+\exp \left[\beta\left(\varepsilon+\left|t_{k}\right|-\mu\right)\right]\right\}^{-1}\right)\right]+1=0 .
\end{aligned}
$$

Here:

$$
n=\frac{n_{1}+n_{2}}{2}, \quad \eta=\frac{\eta_{1}+\eta_{2}}{2}, \quad\left\langle\sigma^{z}\right\rangle=\frac{1}{2} \tanh \left(\frac{\lambda}{2 k T}\right), \quad \lambda=\sqrt{(h+J \eta)^{2}+\Omega^{2}}
$$

At certain conditions, the order of phase transition can change from the second to the first one. The phase transition lines of the first and second order from the uniform phase to the phase with double modulation are shown in figure 1. (a) for different values of the chemical potential $\mu$, (b) for different values of parameter $J$ and for $\Omega=0$ and $\Omega=0.05 \mathrm{eV}$ cases. Transition point of the first kind defined by numerical calculation as the point at which the requirement of minimum for thermodynamic potential with changing the parameters of the model is transition from a homogeneous solution $n_{1}=n_{2}, \eta_{1}=\eta_{2}$ to the modulated with different from zero $\delta n$ and $\delta \eta$. The lines of phase transitions (PT) of the second order are shown bold and the lines of the first order PT are thin. The splitting of the electron band at phase transition is shown in figure 2 The temperature dependences of mean numbers of electrons of sublattice $n_{1}, n_{2}$, and uniform phase $n_{0}$ also, along the phase transition line are shown in figure 3 The temperature dependences of mean values of pseudospins $\eta_{1}, \eta_{2}, \eta_{0}$ are illustrated in figure 3 as well. The temperature dependences of $\delta n(T)$ and $\delta \eta(T)$ are illustrated in figure 4 These results are obtained for the following values of parameters: $g=0.08 \mathrm{eV}, t=0.05 \mathrm{eV}, J=0, \mu=0, \Omega=0$ and $\Omega=0.05 \mathrm{eV}$. The results for $J \neq 0$, $\mu \neq 0$ are presented in figure 1, Such a choice of the parameter values corresponds to the ones given in [1]. All energy characteristics $(T, h, E, g, t, J, \mu, \Omega)$ are in $\mathrm{eV}$ units.

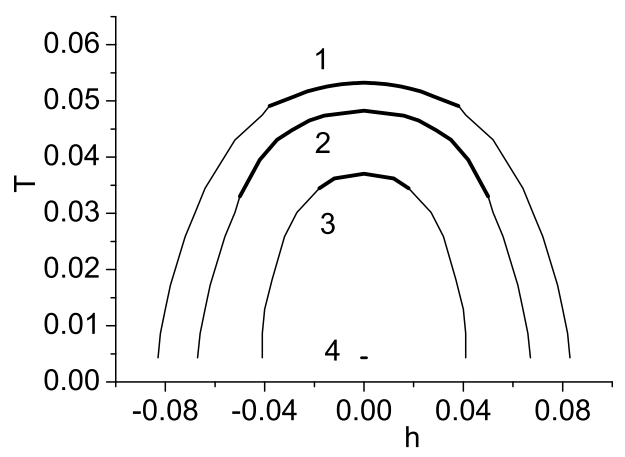

(a)

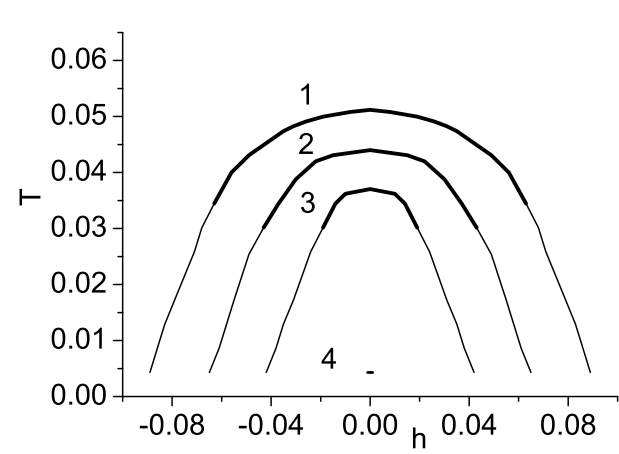

(b)

Figure 1. The phase transition lines of the first and second order from the uniform phase to the phase with double modulation: (a) for different values of the chemical potential $\mu: 1,2,3,4-\mu=0,0.05,0.08,0.12 \mathrm{eV}$ $(\Omega=0, J=0)$, (b) for different values of parameter $J: 1,2,3,4-J=0,0.05,0.1,0.2 \mathrm{eV}(\Omega=0.05 \mathrm{eV}, \mu=0)$. Parameters $T, h$ are in eV units. 


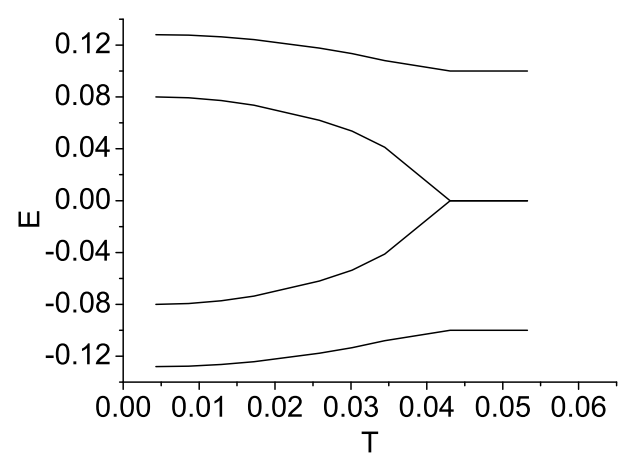

(a)

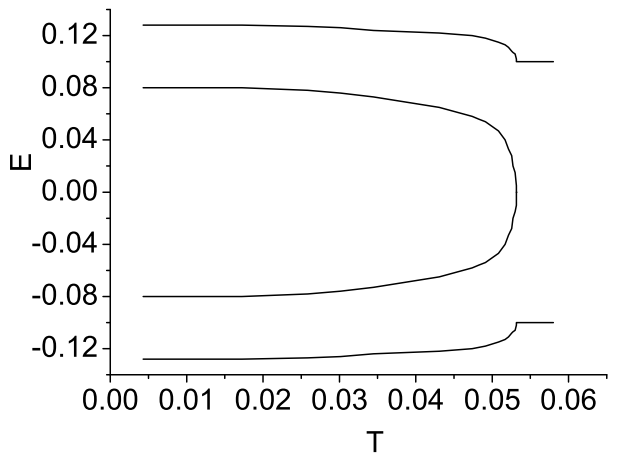

(b)

Figure 2. The splitting of the electron band: (a) along the phase transition line (b) for $h=0$ in charge ordered phase, $\Omega=0$, ( $\mu=0, J=0)$. Parameters $E, T$ are in $\mathrm{eV}$ units.
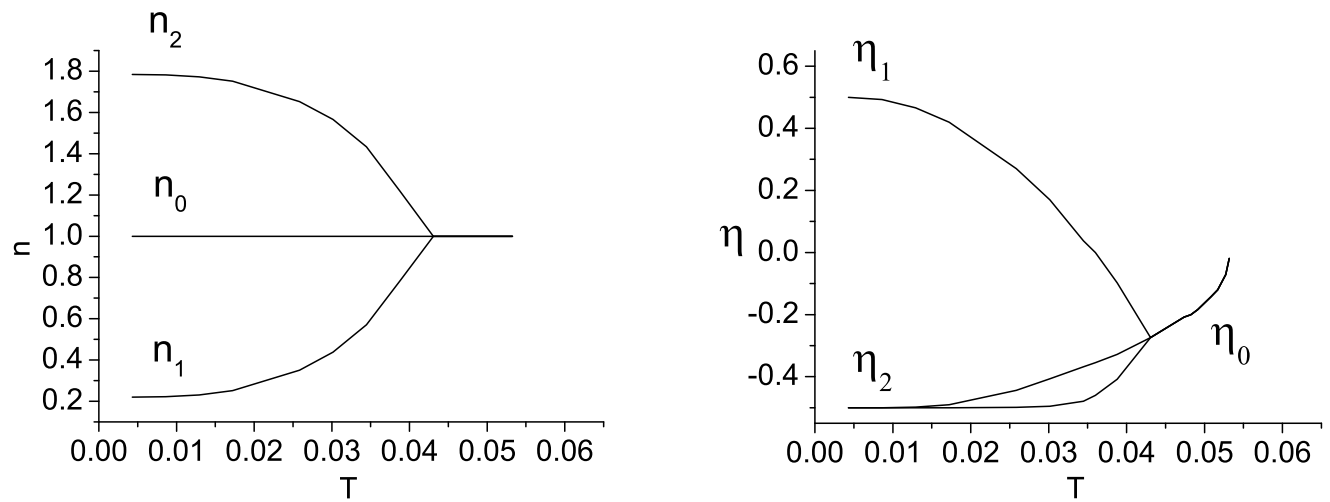

Figure 3. The temperature dependence of mean numbers of electrons of sublattice $n_{1}, n_{2}$, and uniform phase $n_{0}$ and temperature dependence of mean values of pseudospins $\eta_{1}, \eta_{2}, \eta_{0}$ along the phase transition line, $\Omega=0,(\mu=0, J=0)$.
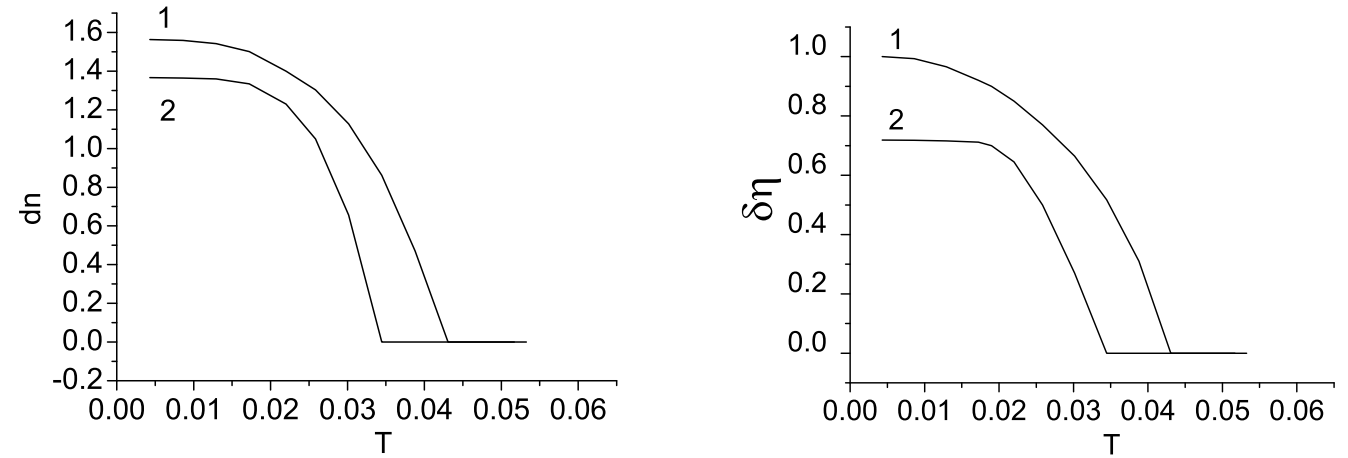

Figure 4. The temperature dependence of the values $\delta n$ and $\delta \eta$ along the phase transition line, $1-\Omega=0$; $2-\Omega=0.05 \mathrm{eV},(\mu=0, J=0)$ 


\section{Dynamic conductivity of quasi-one-dimensional structures with hydrogen bonds}

Calculation of the dynamic conductivity of the structure which possesses the chains with hydrogen bonds was carried out according to Kubo formula [22]

$$
\sigma(\omega, T)=\frac{1}{N a} \int_{0}^{\infty} \mathrm{d} t \exp [\mathrm{i}(\omega+\mathrm{i} \varepsilon) t] \int_{0}^{\beta} \mathrm{d} \lambda\langle\hat{j}(t-\mathrm{i} \hbar \lambda) \hat{j}(0)\rangle,
$$

where $\hat{j}$ is the current density operator

$$
\hat{j}(0)=\frac{\mathrm{i}}{\hbar}[\hat{H}, \hat{d}]
$$

$\hat{d}$ is dipole momentum operator

$$
\hat{d}=(-e) \sum_{l} \sum_{i} R_{i}(l) n_{i}(l)+z_{\mathrm{H}}^{\mathrm{eff}} \delta \sum_{l} \sum_{i} S_{i}^{z}(l),
$$

that includes electronic and pseudospin (ionic) part. Here $\delta$ is the distance between equilibrium positions of a proton on the bond, $\delta \approx 0.40 \AA$. According to quantum-chemical calculations, the effective charge of hydrogen $z_{\mathrm{H}}^{\text {eff }}$ is equal to $z_{\mathrm{H}}^{\text {eff }} \approx 0.25 e$

$$
j(t)=\mathrm{e}^{\frac{\mathrm{i}}{\hbar} H t} j(0) \mathrm{e}^{-\frac{\mathrm{i}}{\hbar} H t} .
$$

In the molecular field approximation, the operator of current density is split into electronic and proton (pseudo-spin) parts

$$
\hat{j}=\hat{j}_{\mathrm{e}}+\hat{j}_{\mathrm{sp}} \text {. }
$$

The following expressions are obtained for these composites:

$$
\begin{aligned}
\hat{j}_{\mathrm{e}}= & -\frac{2 e}{\hbar} \sum_{k, \alpha}\left[\frac{\partial E_{\alpha}(k)}{\partial k_{z}}-(-1)^{\alpha} 2 E_{\alpha}(k) F(k)\right] a_{k, \alpha}^{+} a_{k, \alpha} \\
& +\frac{2 e}{\hbar} \sum_{k} F(k)\left[E_{1}(k)-E_{2}(k)\right]\left(a_{k, 2}^{+} a_{k, 1}+a_{k, 1}^{+} a_{k, 2}\right), \\
\hat{j}_{\mathrm{sp}}= & \frac{\delta}{\hbar} \Omega z_{\mathrm{H}}^{\mathrm{eff}} \sum_{l} \sum_{i, \alpha} S_{i, \alpha}^{y}(l) .
\end{aligned}
$$

Calculation of correlation functions in the expression (10) with the use of the Wick's theorem yields the following expressions for a real part of conductivity:

$$
\sigma=\sigma_{\mathrm{e}}+\sigma_{\mathrm{sp}}
$$

where the electronic part has a form:

$$
\begin{aligned}
\sigma_{\mathrm{e}}(\omega)= & \frac{4 \pi e^{2}}{N a \hbar^{2}} \beta \sum_{k, \alpha}\left[\frac{\partial E_{\alpha}(k)}{\partial k_{z}}-(-1)^{\alpha} 2 E_{\alpha}(k) F(k)\right]^{2} \frac{\mathrm{e}^{\beta\left[E_{\alpha}(k)-\mu\right]}}{\left\{1+\mathrm{e}^{\beta\left[E_{\alpha}(k)-\mu\right]}\right\}^{2}} \delta(\omega) \\
& +\frac{4 \pi e^{2}}{N a \hbar^{2}} \sum_{k} F(k)^{2}\left[E_{2}(k)-E_{1}(k)\right] \frac{\mathrm{e}^{\beta\left[E_{2}(k)-\mu\right]}-\mathrm{e}^{\beta\left[E_{1}(k)-\mu\right]}}{\left\{1+\mathrm{e}^{\beta\left[E_{1}(k)-\mu\right]}\right\}\left\{1+\mathrm{e}^{\beta\left[E_{2}(k)-\mu\right]}\right\}} \\
& \times\left[\delta\left(\omega+\frac{1}{\hbar}\left[E_{2}(k)-E_{1}(k)\right]\right)+\delta\left(\omega-\frac{1}{\hbar}\left[E_{2}(k)-E_{1}(k)\right]\right)\right] .
\end{aligned}
$$

Here

$$
F(k)=-\frac{1}{2} \frac{g\left(\eta_{1}-\eta_{2}\right) t_{k}^{\prime}}{g^{2}\left(\eta_{1}-\eta_{2}\right)^{2}+t_{k}^{2}} .
$$



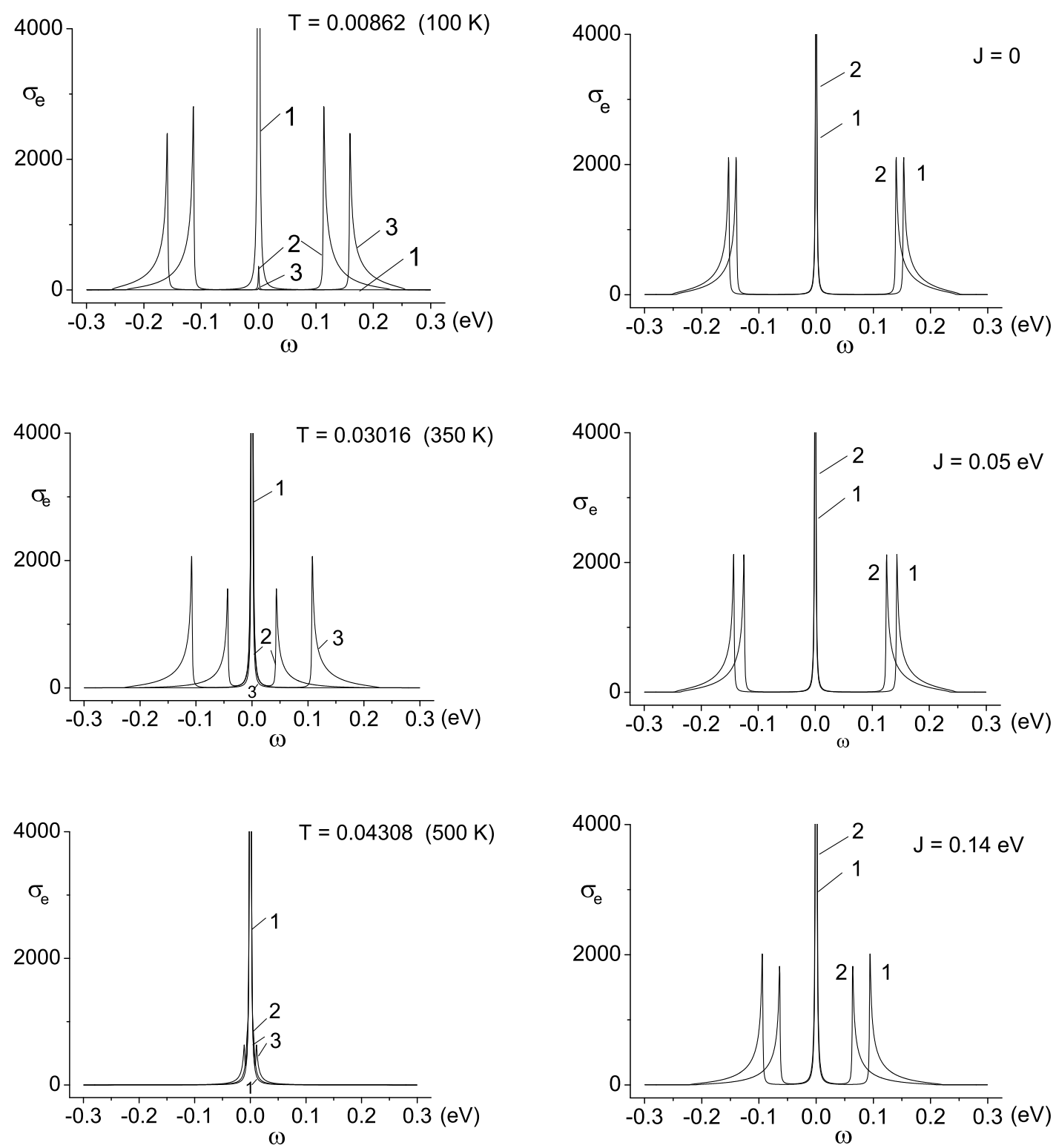

Figure 5. Frequency dependence of the electronic part of conductance: 1 - before phase transition (uniform phase), 2, 3 - after phase transition (modulated structure) along the phase transition line; $2-\Omega=0.05 \mathrm{eV}, 3-\Omega=0 ; \mu=0, J=0$.

Figure 6. Frequency dependence of the electronic part of conductance with different values of proton-proton interaction (parameter $J$ ), $h=0$, $\mu=0, T=0.03016$ (350 K): $J=0 ; 0.05 ; 0.14 ; 1-$ $\Omega=0 ; 2-\Omega=0.05 \mathrm{eV}$.

For the protonic part of conductivity we obtain:

$$
\sigma_{\mathrm{sp}}(\omega)=\frac{\pi}{2 a}\left(\frac{\delta}{2 \hbar} z_{\mathrm{H}}^{\mathrm{eff}} \Omega\right)^{2} \sum_{\alpha} \frac{1}{\lambda_{\alpha}} \frac{1-\mathrm{e}^{-\beta \lambda_{\alpha}}}{1+\mathrm{e}^{-\beta \lambda_{\alpha}}}\left[\delta\left(\omega-\lambda_{\alpha} / \hbar\right)+\delta\left(\omega+\lambda_{\alpha} / \hbar\right)\right] .
$$


Frequency dependence of the electronic part of the dynamical conductivity along the phase transition line is shown in figure 5 curve 1 - before phase transition (uniform phase), curve 2, 3 - after phase transition (modulated structure). At the phase transition from uniform to modulated structure, the conductivity $\sigma_{\mathrm{e}}(0)$, when $\omega=0$, is abruptly reduced by two to three orders of magnitude at low temperatures and with increasing temperature the value of the jump decreases. Electronic conductivity has one peak (at $\omega=0$ ) in uniform phase, (one electronic band is present). We observed the splitting of the electron band in a modulated phase, and electronic conductivity has a broad maximum in the frequency region $\omega=\frac{1}{\hbar}\left[E_{2}(k)-E_{1}(k)\right]$ as well as a peak in $\omega=0$. This broad maximum is placed in the lower frequency regions for structures with the high proton tunneling frequency and stronger direct interaction between protons. The static conductivity $\sigma_{\mathrm{e}}(0)$ in a modulated phase increases with temperature. The dynamical conductivity $\sigma_{\mathrm{e}}(\omega)$ decreases with increasing temperature and its maximum shifts to a lower frequency region and vanishes at the critical temperature when there is no modulation. In a homogeneous phase, only one peak remains at $\omega=0$. The change of the frequency dependence of the dynamical conductivity with the parameters $J$ and $\mu$ is shown in figure 6 and figure 7 (here we consider the case $h=0$ ). Maximum of the dynamical conductivity decreases and shifts to lower frequency region with an increase of $J$ and $\mu$. Critical values of these parameters exist (see figure 1) when the modulated phase vanishes and there remains only a peak at $\omega=0$. The temperature dependence of electronic conductivity $\sigma_{\mathrm{e}}(0)$ is shown in figure 8 , This part of the conductivity is higher for systems with larger proton tunneling frequency and it increases with an increase of the parameters $J$. Conductivity value is presented in relative units.

Proton dynamic conductivity has peaks at frequencies $\omega_{i}=\frac{\lambda_{i}}{\hbar}$ corresponding to protons energies $\lambda_{i}$ on hydrogen bonds. One peak $\left(\lambda_{0}\right)$ exists in case of homogeneous phase and two peaks $\left(\lambda_{1}, \lambda_{2}\right)$ are present for the case of a modulated structure. The dependence of the energy $\lambda_{i}$ on temperature and asymmetry field $h$ along the phase transition line is shown in figure 9
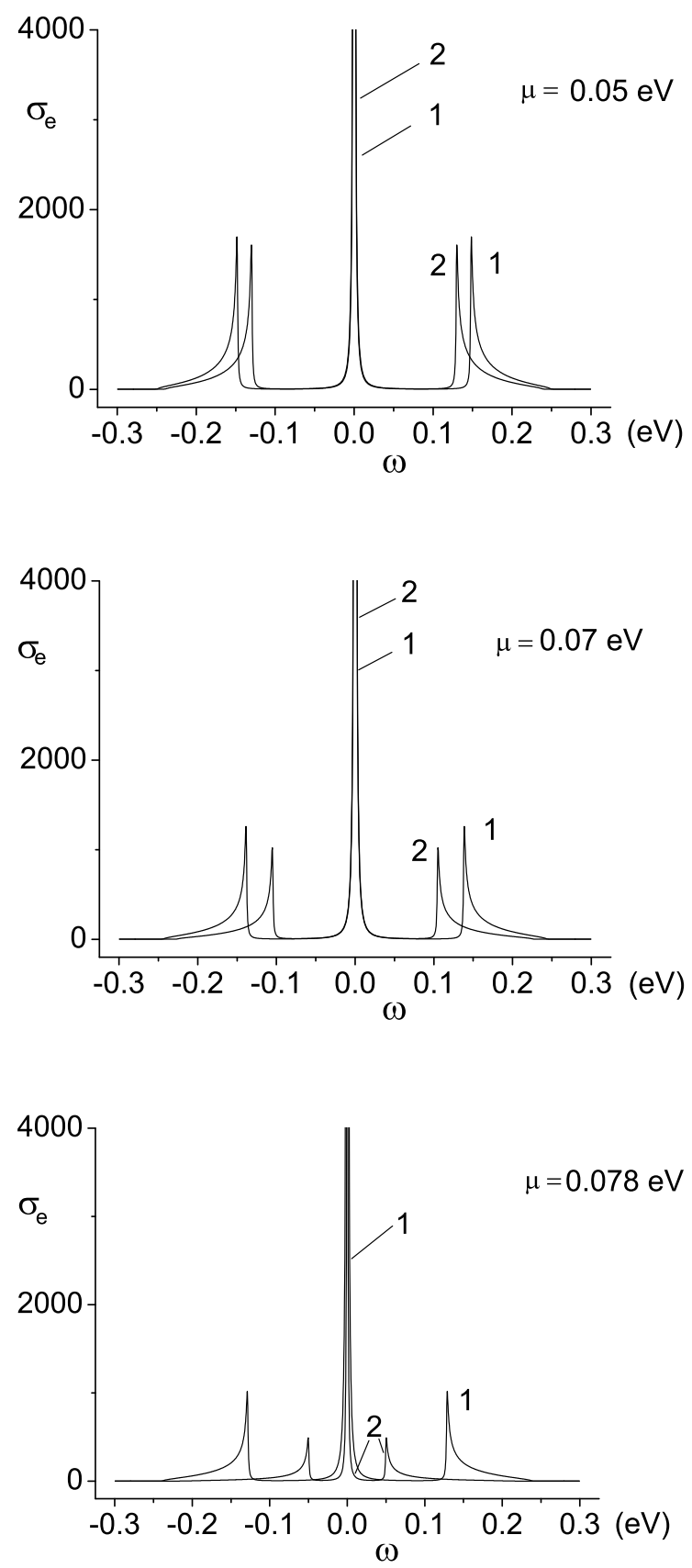

Figure 7. Frequency dependence of the electronic part of conductance with different values of chemical potential $\mu$; $h=0, J=0, T=0.03016$ ( $350 \mathrm{~K}): \mu=0.05 ; 0.07 ; 0.078$; $1-\Omega=0 ; 2-\Omega=0.05 \mathrm{eV}$. 


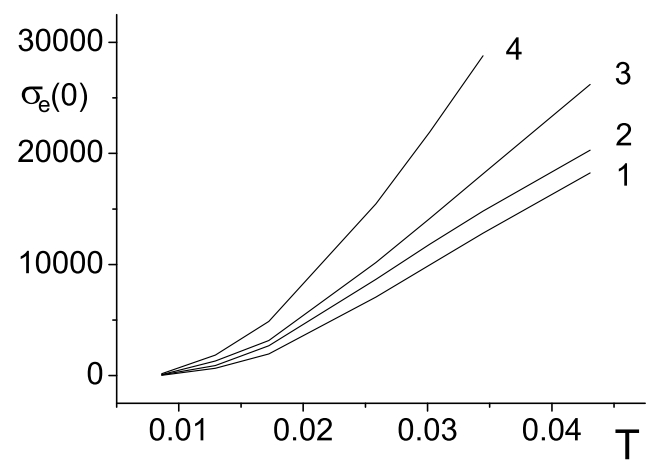

Figure 8. Temperature dependence of the electronic part of conductance of the modulated structure $\sigma_{\mathrm{e}}(0) ; \mu=0 ; h=0 ; 1,2-J=0 ; 3-J=0.05 ; 4-J=0.12 ; 1-\Omega=0 ; 2,3,4-\Omega=0.05 \mathrm{eV}$.
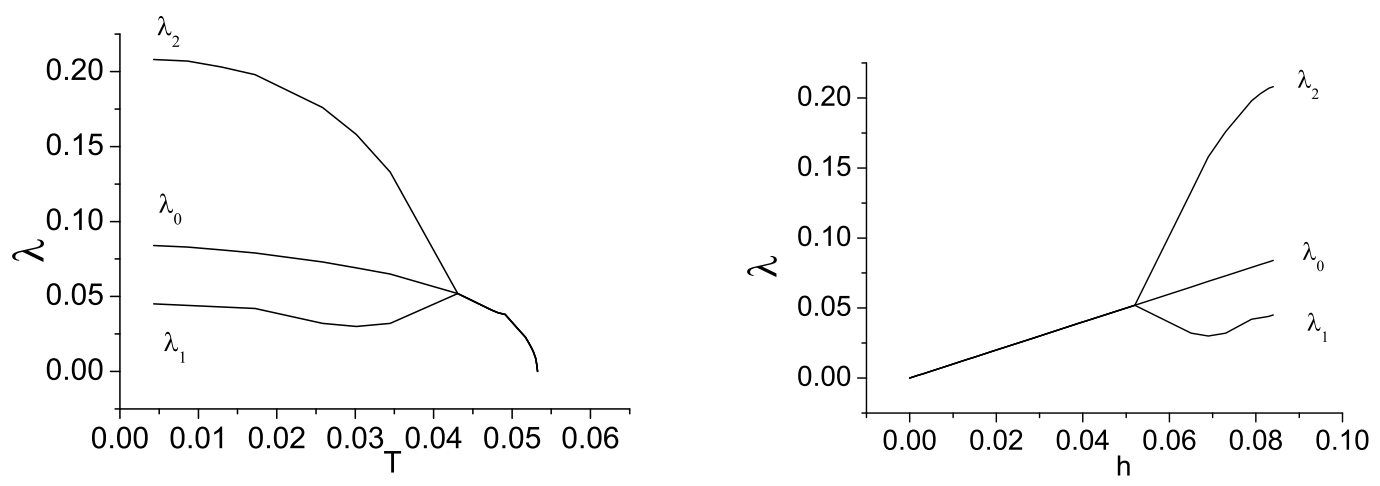

(a)
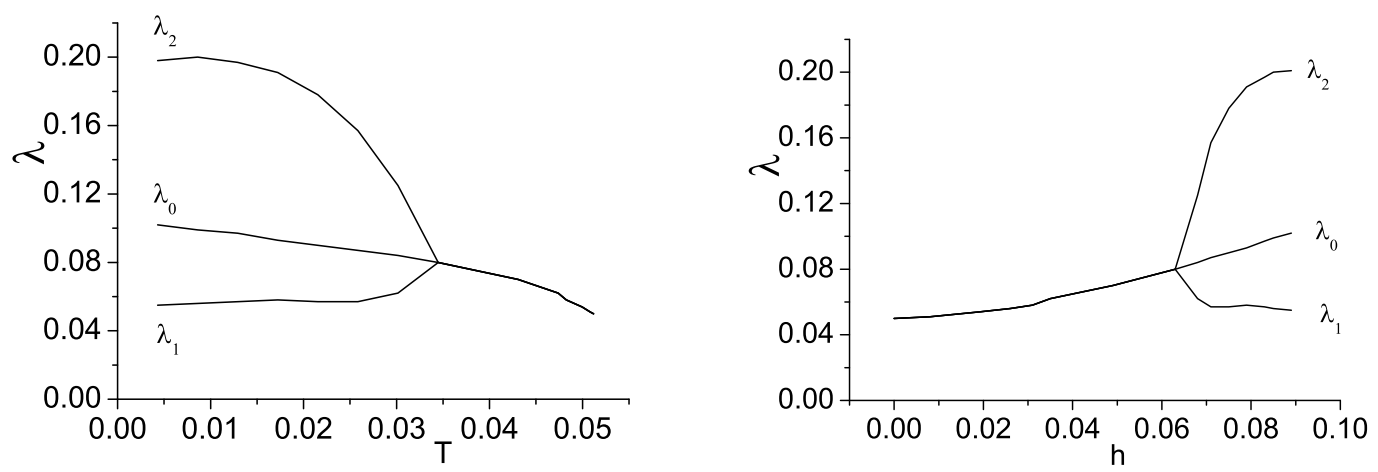

(b)

Figure 9. The dependence of the peak-frequencies of the proton dynamical conductivity on temperature and longitudinal field along the phase transition line; $\lambda_{i}=\hbar \omega_{i} ; \mu=0 ; J=0 ;$ (a) $-\Omega=0$; (b) $-\Omega=0.05 \mathrm{eV}$. 


\section{Conclusions}

The possibility of the first or the second order transitions from uniform phase into phase with doubled lattice period in the quasi-one-dimensional structures with hydrogen bonds is studied in the framework of the proposed pseudospin-electron model. It was shown that pseudospin-electron (proton-electron) interaction may cause the appearance of charge ordered phase in the structures with hydrogen bonds. The electron spectrum is calculated. The dependences of the splitting of the electron spectrum on temperature and asymmetry field are investigated. The dependences of the electron concentration and mean number of protons at the site on temperature and asymmetry field were obtained. It was shown that abrupt changes of these characteristics at the first-order transitions are smaller for the structures with high proton tunneling frequency and stronger direct interaction between protons. The phase transition lines from uniform phase into charge ordered phase are determined. The dependences of the dynamical conductivity on temperature and external field and its changes at the phase transitions are obtained. At the phase transition from uniform to modulated structure the static conductivity $\sigma_{\mathrm{e}}(0)$ is abruptly reduced by two to three orders of magnitude at low temperatures and with increasing temperature the value of the jump decreases. Electronic conductivity has one peak at $\omega=0$ in a uniform phase. In modulated phase, the dynamical electronic conductivity has a broad maximum as well as a peak at $\omega=0$. This broad maximum is placed at lower frequencies for the structures with high proton tunneling frequency and stronger direct interaction between protons. It was shown that the frequency dependence of the proton dynamical conductivity has one peak in a uniform phase and two peaks in the charge modulated phase. The model can be applied to a description of quasi-one-dimensional structures, the so-called halogen-bridge mixedvalence transition-metal complexes [3] in which there are charge modulated states.

\section{References}

1. Stasyuk I.V., Stetsiv R.Ya., Sizonenko Yu.V., Condens. Matter Phys., 2002, 5, 685.

2. Stasyuk I.V., Stetsiv R.Ya., Yurechko R.Ya., J. Phys. Stud., 2005, 9, 349.

3. Okaniwa K., Okamoto H., Mitani T., Toriumi K., Yamashita M., J. Phys. Soc. Jpn., 1991, 60, 997; doi 10.1143/JPSJ.60.997

4. Mitani T., Kitagawa H., Okamoto H., Nakasuji K., Toyota J., Yamashita M., Mol. Cryst. Liq. Cryst., 1992, 216, 73; doi $10.1080 / 10587259208028752$

5. Matsushita N., Toriumi K., Kojima N., Mol. Cryst. Liq. Cryst., 1992, 216, 201; doi 10.1080/10587259208028773

6. Morimoto Y., Tokura Y., Oohashi T., Kojima T., Itsubo A., Mol. Cryst. Liq. Cryst., 1992, 216, 223; doi $10.1080 / 10587259208028777$

7. Okaniwa K., Okamoto H., Mitani T., Inabe T., Tojoda J., Morita Y., Nakasyji K., Yamamoto H., Deno T., Honma S., Mol. Cryst. Liq. Cryst., 1992, 216, 241; doi 10.1080/10587259208028780

8. Takeda S., Chihara H., Inabe T., Mitani T., Maruyama Y., Mol. Cryst. Liq. Cryst., 1992, 216, 235; doi $10.1080 / 10587259208028779$

9. Nakasuji K., Sugiura K., Toyoda J., Morita Y., Okamoto H., Okaniwa K., Mitani T., Mol. Cryst. Liq. Cryst., 1992, 216, 213; doi $10.1080 / 10587259208028775$.

10. Inabe T., Okaniwa K., Okamoto H., Mitani T., Maruyama Y., Takeda S., Mol. Cryst. Liq. Cryst., 1992, 216, 229; doi $10.1080 / 10587259208028778$

11. Stasyuk I.V., Sizonenko Yu.V., Stetsiv R.Ya., J. Phys. Stud., 1998, 2, 463.

12. Hillenbrand E.A., Scheiner S., J. Am. Chem. Soc., 1984, 106, 6266; doi 10.1021/ja00333a027

13. Scheiner S. - In: Proton Transfer in Hydrogen-Bonded Systems. Edited by T. Bountis. Plenum Press, New York, 1992, p. 29-50.

14. Zhao X.G., Cukier R.I., J. Phys. Chem., 1995, 99, 945; doi 10.1021/j100003a017.

15. Cukier R.I., J. Phys. Chem., 1996, 100, 15428; doi 10.1021/jp961025g

16. Fang J.-Y., Hammes-Schiffer S., J. Chem. Phys., 1997, 106, 8442; doi $10.1063 / 1.473903$

17. Fang J.-Y., Hammes-Schiffer S., J. Chem. Phys., 1997, 107, 5727; doi 10.1063/1.474333

18. Matsushita E., Phys. Rev. B, 1995, 51, 17332; doi 10.1103/PhysRevB.51.17332

19. Stasyuk I.V., Shvaika A.M., Tabunshchyk K.V., Condens. Matter Phys., 1999, 2, 109.

20. Stasyuk I.V., Shvaika A.M., Tabunshchyk K.V., Ukr. J. Phys., 2000, 45, 520.

21. Stasyuk I.V., Mysakovych T.S., J. Phys. Stud., 2001, 5, 268.

22. Kubo R., J. Phys. Soc. Jpn., 1957, 12, 570; doi 10.1143/JPSJ.12.570 


\title{
Фазові переходи і динамічні властивості квазіодновимірних структур з водневими зв'язками
}

\author{
Р.Я. Стеців \\ Інститут фізики конденсованих систем НАН України, вул. Свєнціцького, 1, 79011 Львів \\ На основі псевдоспін-електронної моделі досліджено частотну залежність динамічної провідності квазіо- \\ дновимірних систем з водневими зв'язками. В моделі враховано протон-електронну взаємодію, зовнішнє \\ поздовжнє поле $h$, тунелювання протонів, електронне перенесення і пряму протон-протонну взаємодію. \\ Отримано залежність електронної концентрації і середньої заселеності протонних позицій від темпера- \\ тури і поля $h$. Отримано лінію фазових переходів з однорідної фази до фази з модуляцією заряду. Дослі- \\ джено залежність динамічної провідності від поля $h$ і температури та її зміни при фазових переходах.
}

Ключові слова: псевдоспін-електронна модель, протон-електронна взаємодія, водневий зв'язок, провідність 


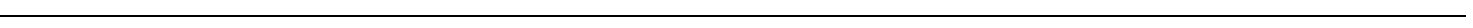

